\title{
DEVELOPMENT OF A MONOLITHIC SHAPE MEMORY ALLOY MANIPULATOR
}

\author{
Kostyantyn Malukhin', Kornel Ehmann ${ }^{2}$ \\ 'Department of Mechanical Engineering, Northwestern University, Evanston, IL, USA 60208 \\ Fax: (847) 4913915 \\ e-mail: k-malukhin@northwestern.edu \\ ${ }^{2}$ Department of Mechanical Engineering, Northwestern University, Evanston, IL, USA 60208 \\ (Adjunct appointments at the University of Illinois at Urbana Champaign, Indian Institute of \\ Technology - Kanpur, India and Chung Yuan Christian University, Chung Li, Taiwan) \\ Tel: (847) 491 3263, fax: (847) 4913915 \\ e-mail: k-ehmann@northwestern.edu
}

\begin{abstract}
The essential steps and issues in the development of a Shape Memory Alloy (SMA) based multi-degrees-of-freedom monolithic manipulator are reflected upon. The use of Direct Metal Deposition (DMD) will be described for the manufacture of the manipulator's structure followed by the exploration of the feasibility of the "self-sensing" functionality of the structure as a basis for the manipulator's control. Finally, a synopsis of a new mathematical model of the kinetics of the temperature-induced phase transformation that was developed and its experimental verification will be given and shown that the analytical predictions closely follow the experimentally measured responses.
\end{abstract}

\section{Introduction}

The paper describes an R\&D effort in progress that is aimed at the development of a compact monolithic meso-scale manipulator for space-limited applications in which a relatively high work output of the manipulator per unit volume of the application is required. Therefore, it is advantageous to use so-called "active" (functional) materials that can simultaneously function as structural as well as actuating and/or sensing elements in the manipulator's embodiment. Active materials include [1]: shape memory alloys, bimetals, piezoelectric materials, magnetostrictors, elecrtostrictors, electrorheological, magnetorheological, thixotropic and rheopex fluids, chemochromic, electrochromic, hydrochromic, photochromic, thermocromic elements, functional gels and others. The use of active materials may potentially result in fewer discrete parts in the manipulator's structure, thus increasing its robustness, precision, controllability, and work output. In addition, according to [2], there are several ways to improve the work output (power transfer to a load) of actuators that use "active" materials, for example, by matching the impedance of the active material and of the load. SMAs can function 
as structural, actuating and sensing elements in an active structure. They function as actuators due to the shape memory effect (SME) property of the alloy, resulting in the ability to recover large, mechanically induced strains [3].

In the current work an active material, namely NiTi (SMA), has been chosen for the fabrication of the proposed meso-scale monolithic manipulator. Three aspects of the development will be addressed. These include: (1) the manufacture and material characterisation of the monolithic manipulator's structure, (2) the feasibility of utilizing the SME to build "self-sensing" SMA structures, and (3) the control of the SMA actuation functions.

For the manufacture of the manipulator, mechanically pre-alloyed NiTi powder was processed by DMD [4] to generate its final geometry. The property of the SMA to undergo temperature-induced phase transformations allows the use of SMA elements as sensors as well. This property was evaluated to assess its feasibility to provide position feedback information. To facilitate the control of the manipulator an analytical modeling effort was undertaken to characterise the kinetics of the phase transformation. Theoretical and experimental results will be presented that confirm the suitability of the model to serve as a basis for the manipulator's control. A synopsis of these three developments is given below.

\section{Design and manufacture of the manipulator}

The conceptual design of the SMA NiTi manipulator is shown in Fig. 1.

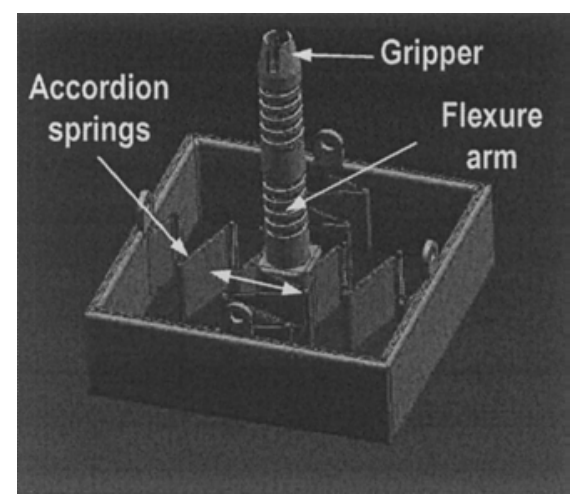

Fig. 1. Conceptual design of the SMA NiTi manipulator: approximate size $40 \times 30 \times 30 \mathrm{~mm}$

The NiTi manipulator is a monolithic one-piece structure, that has three moving parts - accordion springs (accordion spring actuators), flexure arm and a micro- 
gripper. The accordion springs provide the $X-Y$ in-plane motion of the flexure arm. The flexure arm provides the Z-motion of the gripper. All three moving elements posses the shape memory effect (SME), which allows them to generate the necessary motion when heated. The heating of the moving parts can be realized in several ways: (1) direct (resistive) heating, by passing an electrical current through the actuating springs, and (2) indirect heating through external heaters. Due to the relatively high heat capacitance and low thermal conductivity of NiTi alloys (e.g., in comparison to steel), localized heating of the moving elements of the manipulator will not produce a significant heat affected zone.

\section{Characterization of the material fabricated by DMD}

Preliminary research on the quality of the NiTi alloy manufactured by DMD was conducted. Pre-alloyed NiTi powder (NITINOL) was purchased from the Special Metals Corporation. The DMD apparatus was lent to us by Prof. Dr. R. Kovacevic (Southern Methodist University, RCAM lab). DMD is a "layer-by-layer" laser based additive manufacturing process during which metallic powder is deposited onto a substrate. The laser melts the powder, and the solidification of the melt takes place afterwards. It is possible to create complex 2.5- and 3-dimensional metallic structures by laser-based additive manufacturing processes [5].

Several NiTi samples were produced in the form of round plates (Fig. 2). The samples were deposited either on a Ti substrate (Fig. 2a) or on a NiTi (Fig. 2b) substrate. A smaller piece of the sample shown in Figure $2 \mathrm{~b}$ was cut and polished for further analysis of the material's quality and properties. The scanning electron microscope (SEM) image of the polished sample shows a very low amount of pores, no voids, and no cracks (Fig. 3).
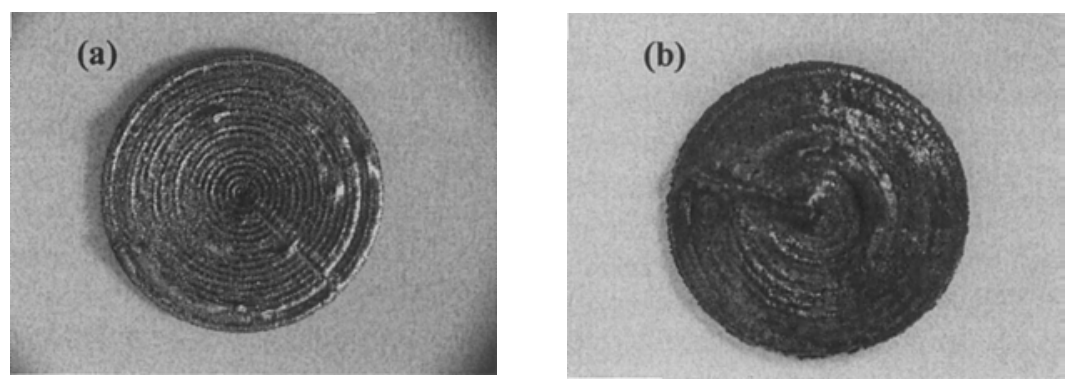

Fig. 2. NiTi samples deposited by DMD: (a) on Ti substrate, (b) on NiTi substrate 


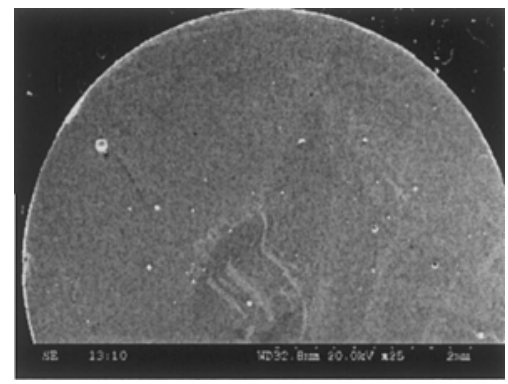

Fig. 3. Scanning electron microscopy (SEM) image of the polished NiTi sample

Secondary ion mass spectroscopy (SIMS) was performed on the sample. The analysis has shown that the amount of impurities in the DMD fabricated NiTi material is lower than in a commercially available NiTi material, manufactured using conventional manufacturing processes.

The DMD fabricated NiTi material was post heat-treated in an oven with a protective Ar atmosphere. Afterwards, the phase transformation properties of the material were measured by differential scanning calorimetry (DSC). The phase transformation temperatures (TTRs) that determine the temperature level at which material transforms from martensite to austenite, and vice versa [6] were measured and found to be close to room temperature. Furthermore, the effect of some of the parameters of the DMD process (laser power, powder feeding rate, and heat sink conditions of the DMD apparatus) was also investigated through a factorial design of the deposition experiments [7]. Several thin-wall structures were deposited onto NiTi substrates with varying DMD parameters (Fig. 4). The responses were the geometrical parameters of the walls and their roughness. Statistical analysis of the results has shown that laser power has an about $60 \%$ influence on the geometric and surface properties of the fabricated thin-walls. It was also found that the intensification of the heat sink conditions will lead to a better control of the quality and the geometry of the NiTi thin-wall structures.

Several prototypes of high quality accordion springs - actuating parts of the manipulator (Fig. 1) - were fabricated [7]. The springs are shown in Fig. 5.

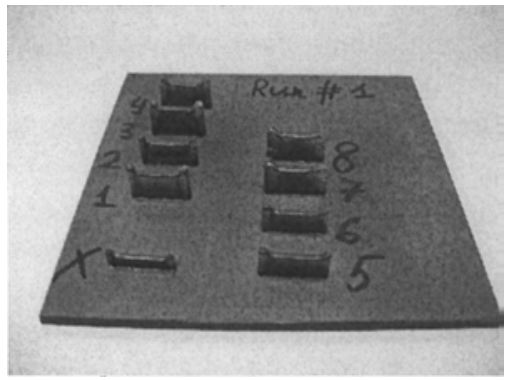

Fig. 4. Thin walls deposited on NiTi substrate

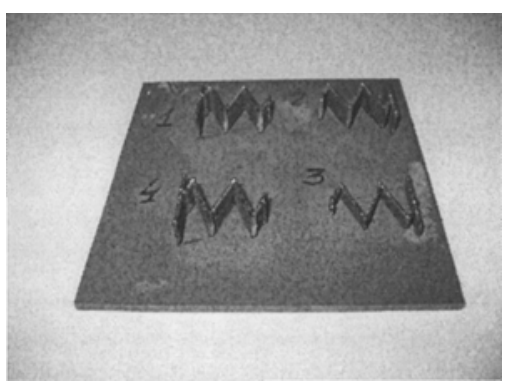

Fig. 5. Accordion springs on NiTi substrate 


\section{4 "Self-sensing" concept}

SMAs can be used as displacement sensors due to the property of the material to change its electrical resistance during heating/cooling. In the present development the possibility of using the structure of the SMA actuator in sensing its motion with a micron level resolution was evaluated. This "self-sensing" property was explored by means of an SMA wire-based actuator [8]. The pre-stretched SMA wire (commercially available Flexinol wire) was subjected to a heat load, allowing the wire to recover its initial non-stretched length due to the shape memory effect. During the recovery process, the change in the electrical resistance of the SMA wire and its contraction displacement were measured. The heat was applied to the wire by passing an electrical current through it. The effect of the heat sink conditions on the response of the SMA wire to the heat load was studied as well. The experimental study has shown that by using the "self-sensing" method it was possible to measure a minimal SMA wire displacement of $17 \mu \mathrm{m}$ with the resolution of $1.7 \mu \mathrm{m}$ as seen from Fig. 6 .

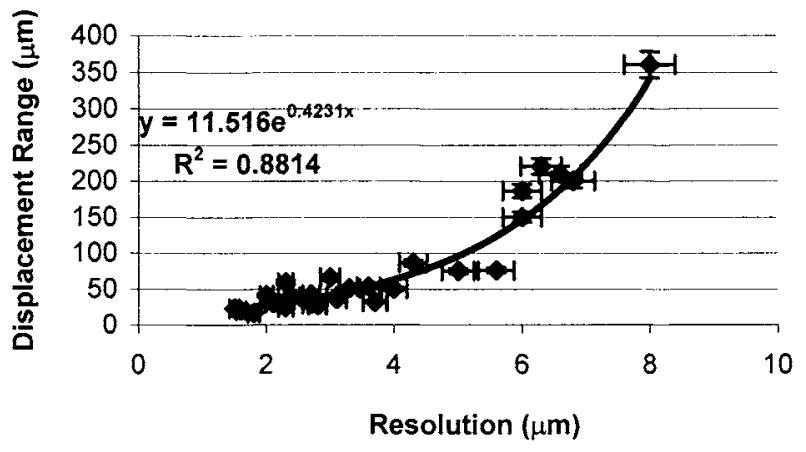

Fig. 6. Resolution of the "self-sensing" method (experimental results)

\section{Mathematical modeling of the kinetics of the phase transformation in SMAs}

A mathematical model describing the kinetics of the phase transformation in the NiTi material subject to a heat load was also developed. The final outcome of the model represents an analytical function of the austenite fraction, $\xi_{A}$, depending on the stress and temperature state in the NiTi material. The purpose of this model was two-fold: (1) to predict the amount of the austenite and, therefore, the magnitude of the recovery displacement of the moving parts of the manipulator during heating [8, 9], and (2) to serve as a basis for the development of the manipulator's controller. The developed model can be expressed as: 
$\xi_{A}=\sqrt{\frac{2 C_{p}}{r_{A}}\left(T-A_{S 0}\right)}$

where:

$C_{P}$ - is the specific heat, $\mathrm{J} /\left(\mathrm{kg}^{\circ} \mathrm{K}\right)$,

$A_{S 0}$ - is the austenite phase transformation start temperature for the stress-free condition,

$r_{A}$ - is the latent heat of the phase transformation in $\mathrm{NiTi}, \mathrm{J} / \mathrm{kg}$.

The phase transformation kinetics model was verified experimentally. NiTi SMA wire actuators were used in the strain recovery experiments conducted. The wires were pre-stretched and heated by resistive heating - electrical current was passed through the wires. The voltage drop across the wires during the heating process was measured and correlated to the amount of the contraction displacement of the SMA wire, generated during the heat induced recovery process. The experimental values of the austenite fraction were calculated as the ratio of the recovered length of the wire to the initial pre-stretched length of the wire. Afterwards, they were compared to the values of the austenite fraction, computed from Eq. 1. The comparison between the experimentally and theoretically obtained values shows a very good agreement (Fig. 7).

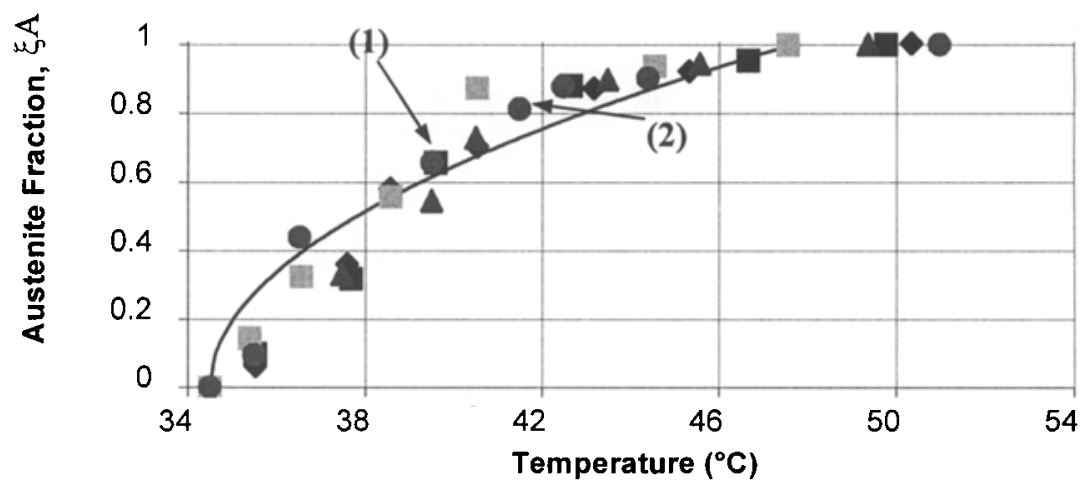

Fig. 7. Experimental (1) and analytical (2) values of the austenite fraction evolution during temperature-induced phase transformation 


\section{Mathematical modeling of the linear motion of the manipulator}

The suggested phase transformation model serves as a basis for the development of the control law of the motion of a NiTi SMA manipulator. Firstly, the motion model of the linear stage of the manipulator was developed based on the equations of motion written for an "agonistic-antagonistic" layout of the accordion spring actuator according to $[8,9,10]$. Step response experiments were carried out using an SMA NiTi wire. The numerical simulations of the step response of the model of the motion of the "agonist-antagonist" actuator were undertaken using the "Simulink" toolbox in "MatLab" environment. The comparison of the simulation and experimental results is shown in Fig. 8.

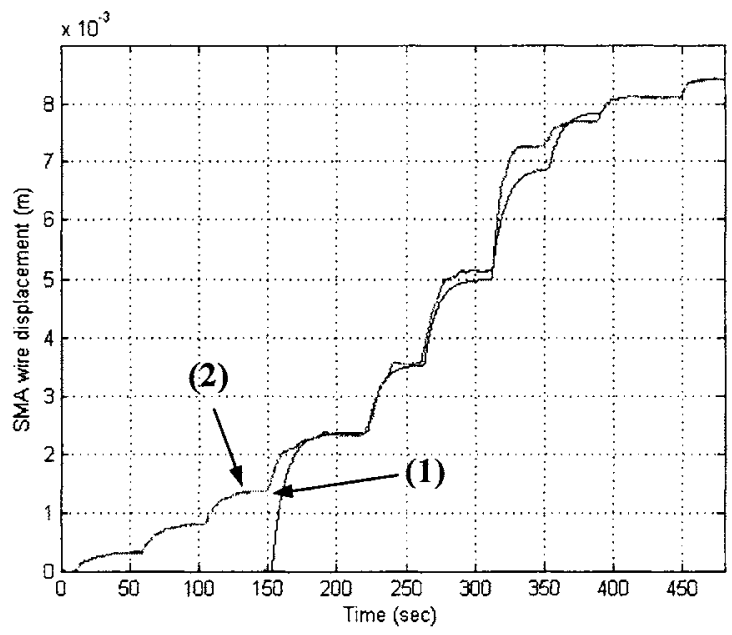

Fig. 8. Comparison of the numerical simulation (1) and experimental results (2) of the step response of the "agonist-antagonist" actuator model

Figure 8 shows an excellent agreement between the modeled and the experimentally measured displacements of the SMA wire-based actuator. Currently, work is in progress on applying this model to the accordion spring-based actuator.

\section{Conclusions}

The DMD process was used to process NiTi powder into high quality (non-porous, non-cracked, low contamination level) structures. A factorial design of experiments was used to identify optimal parameters for the deposition of thin walls by DMD from NiTi powder. It was possible to DMD fabricate several a high quality thinwall NiTi structures - accordion springs. A mathematical model of the kinetics of temperature-induced phase transformation was developed and experimentally 
verified. A model of motion of an SMA actuator, based on the kinetics model, was developed and experimentally verified. The model will be used in the development of a control model of motion of arbitrary SMA actuators.

\section{Acknowledgments}

We are thankful to Professor Dr. R. Kovacevic and his colleagues at Southern Methodist University/RCAM for giving us the opportunity to use the DMD equipment and for their valuable help in all the DMD experiments. We also acknowledge the financial support of the National Science Foundation (USA) under grant \#DMI-0400316.

\section{References}

1. H. Janocha, Actuators: basics and applications (Springer Berlin Heidelberg, New York 2004).

2. G.A. Lesieutre, J. Loverich, G.H. Koopmann, and E.M. Mockenstrum, Increasing the Mechanical Work Output of an Active Material Using a Nonlinear Motion Transmission Mechanism, Journal of Intelligent Material Systems and Structures, 15, pp. $49-58$ (2004).

3. L.C Brinson, One-Dimensional Constitutive Behavior of Shape Memory Alloys: Thermomechanical Derivation With Non-Constant Material Functions and Redefined Martensite Internal Variable, Journal of Intelligent Materials Systems and Structures 4 (2), pp. 229-242 (1993).

4. R. Kovacevic R (2007); http://engr.smu.edu/rcam/rcamweb/index.htm.

5. J.K. Wessel. Handbook of advanced materials: enabling new designs (J. Wiley, Hoboken, N.J. 2004).

6. K. Malukhin and K. Ehmann, K., Material Characterization of NiTi Based Shape Memory Alloys Fabricated by the Laser Direct Metal Deposition Process, Journal of Manufacturing Science and Engineering, 128, pp. 691-696 (2006).

7. K. Malukhin, K. Ehmann, Identification of Direct Metal Deposition (DMD) Process Parameters for Manufacturing Thin Wall Structures from Shape Memory Alloy (NiTi) Powder, Transactions of the North American Manufacturing Research Institution of SME, 35 (2007).

8. K. Malukhin, K. Ehmann, The Possibility of the Development of a "Self-Sensing" Shape Memory Alloy Based Actuator, Proceedings of the 1st International Conference on Micromanufacturing ICOMM 2006, pp. 231-236 (2006).

9. K. Malukhin and K. Ehmann, Model of Motion of an Actuator Based on a NiTi Shape Memory Alloy. Proceedings of the $2^{\text {nd }}$ International Conference on Micromanufacturing ICOMM 2007, (2007).

10. C.H. Wu, J.C. Houk, K.-Y. Young, L.E. Miller in: Nonlinear Damping of Limb Motion/Multiple Muscle Systems: Biomechanics and Movement Organization, edited by J.M.. Winters and S.L-Y Woo, (Springer-Verlag, New York 1990). 